\title{
Editorial: ROS Regulation during Plant Abiotic Stress Responses
}

\author{
Zhulong Chan ${ }^{1 * t}$, Ken Yokawa ${ }^{2,3+}$, Woe-Yeon $\mathrm{Kim}^{4 \dagger}$ and Chun-Peng Song ${ }^{5 * t}$ \\ ${ }^{1}$ Key Laboratory of Plant Germplasm Enhancement and Specialty Agriculture, Wuhan Botanical Garden/Sino-Africa Joint \\ Research Center, Chinese Academy of Sciences, Wuhan, China, ${ }^{2}$ Institute of Cellular and Molecular Botany, University of \\ Bonn, Bonn, Germany, ${ }^{3}$ Department of Biological Sciences, Tokyo Metropolitan University, Tokyo, Japan, ${ }^{4}$ Division of \\ Applied Life Science (BK21 Plus), Plant Molecular Biology and Biotechnology Research Center, Institute of Agriculture and \\ Life Sciences, Gyeongsang National University, Jinju, South Korea, ${ }^{5}$ Department of Biology, Henan University, Kaifeng, China
}

Keywords: abiotic stress, hormones, reactive oxygen species, redox

\section{The Editorial on the Research Topic}

\section{ROS Regulation during Plant Abiotic Stress Responses}

Plants frequently encounter a combination of abiotic stresses in their natural habitats. Abiotic stresses, including drought, salt, cold, heat, and heavy metal etc., modulate phytohormone metabolism and enhance expression level of transcription factors which activate stress responsive genes. During plant stress response, reactive oxygen species (ROS) act as important molecules and play pivotal roles in activating downstream metabolic pathways. In this Research Topic, we collected 25 manuscripts related to ROS and redox regulation in plant responses to abiotic stress, including reviews of the role of ROS in plant abiotic stress responses and articles related to gene function analysis, genome-wide gene expression and transcriptomic analysis, and interaction analysis between ROS and phytohormones.

Krieger-Liszkay and Feilke reviewed how plastid terminal oxidase (PTOX) interplays with the photosynthetic electron flow and hypothesized that the function of PTOX is dependent of stromal pH. Corpas and Barroso briefly summarized possible roles of reactive sulfur species (RSS) in peroxisomes and hypothesized potential interactions among ROS, reactive nitrogen species (RNS) and sulfur-containing compounds. In crop plants, ROS regulation under abiotic stress condition has been reviewed by You and Chan. In response to abiotic stress, plants have evolved complex signaling pathways to regulate sets of stress responsive genes encoding protein kinases, phosphatases, transcriptional factors, SIMILAR TO RCD ONE (SRO) proteins, ROS-scavenging or detoxification proteins, and proteins involved in hormone pathway and calcium signal (You and Chan). Sewelam et al. summarized that ROS functions as the primary source of the signaling battery in plants under stressed conditions and may interact with other signaling components, e.g., calcium, redox homeostasis, membranes, G-proteins, MAPKs, plant hormones, and transcription factors. Sewelam et al. discussed the interaction between nitric oxide (NO) and ROS which might regulate abscisic acid (ABA) biosynthesis to modulate stomatal closure. Liu et al. reviewed the functions of polyamines (PAs) during plant stress response through modulation of antioxidant systems or suppression of ROS production. Evidences showed that PA catabolism resulted in the production of $\mathrm{H}_{2} \mathrm{O}_{2}$. Exogenous supply of PAs or ectopic expression of PAs biosynthesis related genes increased antioxidant system in several plants (Liu et al.). Dinakar et al. demonstrated the importance of AOX pathway in optimizing photosynthesis in Pisum sativum in the presence of osmotic and temperature stress conditions. These articles summarized roles of ROS during plant stress response and possible interaction of ROS with hormones and other chemicals.

Compared to extensive studies on drought, cold, heat, and osmotic stresses, less attention has been paid to heavy metal stress and light stress, which become increasingly important stress factors 
limiting plant growth. Three research articles addressed aluminum (Al), cadmium $(\mathrm{Cd})$ and UV-B stress responses, respectively. Lin et al. observed that $\mathrm{Al}^{3+}$ stress induced $\mathrm{O}_{2}^{-\bullet}$ generation in the cell suspension cultures of tobacco and rice, while pretreatment with various concentration of $\mathrm{Zn}^{2+}$ significantly inhibited the $\mathrm{Al}^{3+}$-induced oxidative burst. Moreover, $\mathrm{Al}^{3+}$-induced cell death was also inhibited in the presence of $\mathrm{Zn}^{2+}$. High concentration of zinc $(0.5 \mathrm{mM})$ effectively lowered the level of $\mathrm{Al}^{3+}$-induced $\left[\mathrm{Ca}^{2+}\right]_{c}$ elevation. In tomato, cadmium (Cd) stress significantly inhibited activities of superoxide dismutase (SOD), catalase (CAT) and peroxidase (POD), while increased the contents of $\mathrm{H}_{2} \mathrm{O}_{2}$ and $\mathrm{O}_{2}^{-\bullet}$, resulted in increased malondialdehyde (MDA) and electrolyte leakage (EL) (Hasan et al.). Yokawa et al. found that UV-B promoted the robust generation of ROS which affects endocytic vesicle recycling in Arabidopsis root apex. It is well known that many root tropisms require elaborate control of endocytic vesicle recycling in the cells. This finding explains that how light stress situation triggers root negative phototropism through ROS production as a rapid signaling event.

Omic approaches were effectively applied to identify genes involved in plant stress responses. de Abreu Neto and Frei conducted a meta-analysis of microarray experiments in rice. Publicly available microarray transcriptome data were re-analyzed. The results showed that ROS-related genes were overrepresented among the differentially expressed genes (DEGs). After treatments with oxidative stress (ozone and $\mathrm{H}_{2} \mathrm{O}_{2}$ ) and abiotic stresses, 990 and 1727 shared DEGs were identified, respectively. Among them, 311 genes were overlapped by both oxidative and abiotic stresses and 33 were ROS-related genes. Additionally, Mata-Pérez et al. identified genes in response to linolenic acid, a precursor of jasmonic acid (JA) using RNAseq approach. In total, expression levels of 3034 genes were changed after linolenic acid treatment. This study showed that linolenic acid modulated the expression of genes involved in stress response, particularly those mediated by ROS signaling. Several transcription factors including WRKY, JAZ, MYC were also modified in response to linolenic acid. These data indicated that abiotic stress modulated expression of stress responsive genes as well as ROS related genes. Wei et al. identified 85 WRKY genes in cassava (Manihot esculenta) through bioinformatics analysis. RNA-seq data showed that $78 \mathrm{MeWRKY}$ genes were differentially expressed in response to drought stress and 9 $M e W R K Y$ genes were modulated after $\mathrm{NaCl}$, mannitol, cold, $\mathrm{H}_{2} \mathrm{O}_{2}$ and $\mathrm{ABA}$ treatments, indicating that $M e W R K Y$ genes were involved in plant stress response and redox signaling pathway. These analyses provided new clues for identification of genes involved in oxidative stress.

Detailed functions of several stress responsive genes including transcription factors have been characterized. The Universal Stress Protein domain (USP) gene modulated plant response to a wide variety of abiotic stresses. The biochemical function of AtUSP (At3g53990) was characterized by Jung et al. The results showed that AtUSP-OX plants were tolerant to heat shock and oxidative stresses, whereas the knock-out mutants were sensitive to the stress treatments. AtUSP exhibited a redox-dependent chaperone function which might contribute to its protective roles during diverse stress conditions. The Arabidopsis sulfotransferase gene AtSOT12 is a salt inducible gene through transcriptome analysis. Chen et al. found that salt stress affected capping and polyadenylation of AtSOT12, but not DNA methylation level in the promoter region. Expression of AtSOT12 was induced by salt stress is partially through ABA-INSENSITIVE 1 (ABI1)-and SALT OVERLY SENSITIVE 1 (SOS1)-mediated signaling pathways. Mutation of oxidative stress related oxi1 resulted in increased AtSOT12 expression, while ROS scavenger treatments also enhanced AtSOT12 transcript level, indicating that ROS production might be involved in the repression of AtSOT12 gene. Baek et al. isolated an Arabidopsis ars1 (aba and ros sensitive 1) mutant which showed hypersensitivity to ABA and methyl viologen (MV). ARS1 encodes a nuclear protein with one zinc finger domain. Expression level of CSD3 gene encoding SOD was reduced and ROS was accumulated in ars1 mutant. Furthermore, ARS1 inhibited ABA-induced ROS production. Treatment with ABA, $\mathrm{H}_{2} \mathrm{O}_{2}$ and $\mathrm{MV}$ modulated localization of ARS1 protein. Wang et al. assembled 10 WRKY unigenes from ESTs of wheat (Triticum aestivum). Among them, TaWRKY44 was upregulated by various stress treatments, hormones, and $\mathrm{H}_{2} \mathrm{O}_{2}$. TaWRKY44 localizes to the nucleus and binds to the core DNA sequences of TTGACC and TTAACC in yeast. TaWRKY44 transgenic tobacco plants showed increased drought and salt tolerance. Under osmotic stress condition, transgenic lines exhibited lower $\mathrm{H}_{2} \mathrm{O}_{2}$ content and higher SOD, CAT, and POD activities. Overexpression of TaWRKY44 increased expression of several ROS related genes and stress-responsive genes. The results indicated that several transcription factors might function as ROS upstream regulators.

In response to environmental stresses, plants develop various strategies, including induction of phytohormones. Among the, auxin regulates plant growth and development. YUCCA6, a flavin monooxygenase enzyme, converts indole-3-pyruvic acid to auxin. Cha et al. reported that overexpression of YUCCA6 in Arabidopsis reduced the expression of senescence related gene SAG12 and delayed leaf senescence. YUCCA6-OX plants, but not mutated YUCCA6-OX ${ }^{\mathrm{C} 85 \mathrm{~S}}$, a dysfunctional mutation of ROS homeostasis maintained by YUCCA6, exhibited reduced ROS accumulation and increased expression of genes encoding NADPH-dependent thioredoxin reductases and GSH1 involved in redox signaling. Moreover, auxin efflux proteins at both transcriptional and protein level were reduced either by ROS balance or by thiol-reductase activity of YUCCA6. Additionally, overexpression of the cytokinin biosynthetic gene AtIPT8 (adenosine phosphate-isopentenyltransferase 8) in Arabidopsis thaliana resulted in increased endogenous cytokinin content (Wang et al.). AtIPT8 transgenic lines showed increase sensitivity to salt stress and accumulated higher ROS content than the wild type control. Moreover, many genes involving in photosynthesis and abiotic stress responses were differentially expressed in AtIPT8 transgenic lines (Wang et al.). Therefore, phytohormones affected plant stress responses partially through modulation of ROS levels.

Exogenous application of hormones and chemicals increased plant tolerance to various abiotic stresses. Treatment with spermidine promoted the growth recovery of rice after drainage. 
Spermidine treatment decreased ROS generation and improved photosynthesis in submerged rice. Addition of polyamine (PA) alleviated the suppressing effects of osmotic stress in leaves of white clover. Further study showed that PA was involved in regulation of $\mathrm{H}_{2} \mathrm{O}_{2}$ and $\mathrm{Ca}^{2+}$ messenger. PAinduced $\mathrm{H}_{2} \mathrm{O}_{2}$ production required $\mathrm{Ca}^{2+}$ release, while PAinduced $\mathrm{Ca}^{2+}$ release was also essential for $\mathrm{H}_{2} \mathrm{O}_{2}$ production (Li et al.). Pretreatment with spermidine in rice improved submergence tolerance through modulation of ROS production and chlorophyll degradation (Liu et al.). Xu et al. found that exogenous ascorbic acid treatment improved root growth in tall fescue (Festuca arundinacea) under water stress condition. Roots in ascorbic acid (ASA)-treated plants had lower ROS and MDA contents, higher non-enzymatic antioxidant accumulation, and increased expression of genes encoding cell-wall loosening proteins. Melatonin (N-acetyl-5-methoxytryptamine) has long been known to be an important animal hormone and identified in various plant species since 1995. In tomato (Solanum lycopersicum), cadmium (Cd) stress significantly increased the contents of $\mathrm{Cd}$ and melatonin (Hasan et al.). Exogenous application of melatonin increased activities of antioxidant enzymes and $\mathrm{H}^{+}$-ATPase, and contents of glutathione (GSH) and phytochelatins. Supplementation with melatonin significantly reduced leaf $\mathrm{Cd}$ accumulation. In common wheat (Triticum aestivum), application of ABA caused decreased contents of $\mathrm{H}_{2} \mathrm{O}_{2}$ and MDA and increased GSH and ASA under osmotic stress condition (Wei et al.). Gene expression analysis showed that ABA treatment regulated transcripts of genes encoding ASA and GSH synthesis-related enzymes. The results shed lights on exogenous application of chemicals to improve plant stress tolerance.

Under abiotic stress condition, metabolites like dehydrin and proline appear to function in stress tolerance by serving as a compatible solute or osmoprotectant. Dehydrin belongs to group II late embryogenesis abundant protein (LEA). Shi et al. characterized functions of Arabidopsis LOW TEMPERATURE-INDUCED 30 (LTI30), encoding a LEA protein, under drought stress condition. AtLTI30 knockout mutant was less sensitive to $\mathrm{ABA}$ and displayed decreased drought tolerance, whereas AtLTI30-OX plants were more sensitive to ABA and showed improved drought tolerance. Manipulation of AtLTI30 expression increased activities of CAT and decreased drought stress-triggered $\mathrm{H}_{2} \mathrm{O}_{2}$ production. In trifoliate orange (Poncirus trifoliate), Peng et al. cloned a hybrid proline-rich protein gene PtrPRP. Expression level of PtrPRP was progressively induced upon cold stress treatment. PtrPRP knock-down lines displayed sensitivity to cold stress as evidenced by higher EL, MDA content, and increased accumulation of ROS. In aged oat seed, Kong et al. suggested that proline and antioxidant enzymes played the main role in adaptation to oxidative stress in seeds with higher $(28 \%)$ and lower $(4 \%, 16 \%)$ moisture contents, respectively. These studies highlighted the protective roles of osmoprotectants and putative functions in modulating of ROS and redox pathways.

In summary, we focus on the roles of ROS during plant abiotic stress responses in this Research Topic. Plant responses to multiple abiotic stresses and effects of hormones and chemicals on plant stress responses have been carefully studies. Although functions of several stress responsive genes have been characterized and possible interactions between hormones and ROS are discussed, future researches are needed to functionally characterize ROS regulatory and signaling transduction pathways.

\section{AUTHOR CONTRIBUTIONS}

ZC and CS wrote the manuscript, KY and WK added notes and revised the manuscript.

Conflict of Interest Statement: The authors declare that the research was conducted in the absence of any commercial or financial relationships that could be construed as a potential conflict of interest.

Copyright (c) 2016 Chan, Yokawa, Kim and Song. This is an open-access article distributed under the terms of the Creative Commons Attribution License (CC BY). The use, distribution or reproduction in other forums is permitted, provided the original author(s) or licensor are credited and that the original publication in this journal is cited, in accordance with accepted academic practice. No use, distribution or reproduction is permitted which does not comply with these terms. 